\title{
Cryptococcosis in apparently immune-competent patients: taxonomy, epidemiology, pathophysiology and treatment
}

\author{
Rachel Wake ${ }^{\mathrm{a}, \mathrm{b}}$ and Nelesh P Govender ${ }^{\mathrm{a}, \mathrm{c} *}$ \\ ${ }^{a}$ National Institute for Communicable Diseases [Centre for Opportunistic, Tropical and Hospital Infections], Johannesburg, South Africa \\ 'Institute for Infection and Immunity, St. George's University of London, London, UK \\ 'Division of Medical Microbiology, Faculty of Health Sciences, University of Cape Town, Cape Town, South Africa \\ *Corresponding author, email: Neleshg@nicd.ac.za
}

\begin{abstract}
Taxonomy
Cryptococcosis, a systemic mycosis with a worldwide distribution, is caused by fungi within the pathogenic Cryptococcus neoformans-Cryptococcus gattii species complex. Until recently, this species complex simply included two species, namely $C$. neoformans (containing serotypes A [C. neoformans var. grubii], D [C. neoformans var. neoformans], and an AD hybrid) and C. gattii (containing serotypes $B$ and $C$ ). In 2015, the current $C$. neoformans var. grubii and C. neoformans var. neoformans were proposed to be recognised as separate species, and five separate species were proposed within C. gattii, in addition to several hybrid species. ' While this revised nomenclature has taxonomic relevance, it is unlikely to filter down to the clinic, not least because the molecular tools to separate these cryptic species are rarely available at diagnostic laboratories.
\end{abstract}

\section{Epidemiology}

Largely fuelled by the AIDS pandemic, the global burden of cryptococcosis is enormous, with a recent estimate of 221400 (95\% Cl 180640 to 267600 ) cases per annum, 70\% of which occur in sub-Saharan Africa (155 030 cases, 95\% Cl 126950 to 186 240). ${ }^{2}$ By country, Nigeria, India and South Africa are estimated to have the highest number of cases. Cryptococcus neoformans var. grubii [proposed name: $C$. neoformans] is the most common cause of meningitis among HIV-seropositive adults in southern and eastern Africa. ${ }^{3,4}$ Cryptococcosis rarely occurs in HIV-seronegative individuals (around $1 \%$ of cases in South Africa). ${ }^{5}$ Although most patients have a known risk factor, suchas solidorgantransplantorcell-mediated immunodeficiency, around $10 \%-40 \%$ are apparently immune-competent. ${ }^{6,7}$ Disease in immune-competent patients is commonly caused by C. gattii species-complex; is more likely to have extra-neural and extrapulmonary manifestations; and, is associated with a higher risk of mortality. ${ }^{6-9}$

Paediatric cryptococcosis is relatively less common than adult disease: 1 case per 100000 people vs 19 cases per 100000 people in the general population. ${ }^{10}$

The case reported in this edition of the Journal is, therefore, unusual in several respects: disseminated disease (involving the lungs, blood, bone marrow, skin, meninges, urinary tract, liver and lymph nodes) caused by C. neoformans var. neoformans [proposed name: $C$. deneoformans], which occurred in a young child who was considered to be immune-competent.

\section{Pathophysiology}

Since Cryptococcus is a ubiquitous fungus, it is unsurprising that exposure, through inhalation of spores or desiccated yeast cells is likely to be almost universal during early childhood. Serological studies reveal that children acquire antibodies to $C$. neoformans from the age of two years, ${ }^{11}$ and that infection is usually asymptomatic in both immune-compromised and immunecompetent individuals. ${ }^{11-13}$

Following inhalation, Cryptococcus is thought to remain dormant in the body. Evidence of latency exists in the finding of continuous antibody production, ${ }^{11-14}$ molecular studies indicating that cryptococcosis presenting in Africans living in Europe was acquired many years earlier, ${ }^{15,16}$ and in autopsy studies from patients without a history of cryptococcal disease or exposure describing pulmonary granulomas containing C. neoformans. ${ }^{17,18}$ The ability of the fungus to persist in humans without triggering a successful immune reaction has likely evolved due to its saprophytic nature (reviewed in ${ }^{7-9}$ ). For example, C. neoformans is able to survive and replicate within macrophages in a similar way to which it exists as an intracellular parasite of amoebae in the environment. ${ }^{19,20}$ Furthermore, its polysaccharide capsule which protects it from harsh conditions, including UV light and extremes of temperature in the environment, exhibits antiphagocytic mechanisms, causing macrophage dysfunction and lysis, and allowing resistance to phagosomal digestion. ${ }^{21}$ These, along with many other 'ready-made' virulence factors (production of melanin, degradative enzymes and an ability to grow at physiological temperatures), ${ }^{22}$ allow Cryptococcus to remain in dormant until host immune-compromise allows dissemination and disease, most commonly meningoencephalitis in HIV-infected individuals.

Although disease usually represents reactivation of latent infection in immune-compromised hosts, primary acquisition can also be associated with clinical symptoms. ${ }^{23}$ Acute infection may be more likely following exposure to a greater number of Cryptococcus cells. ${ }^{24}$ This may be the mechanism of cryptococcal disease described in this case.

The rare occurrence of cryptococcosis in apparentlyimmunocompetent hosts highlights the multiplicity of innate and acquired immune factors, and complex immune interactions, required for successful defence against $C$. neoformans. Although $\mathrm{CD} 4^{+} \mathrm{T}$-cell function is clearly of key importance (as demonstrated by the emergence of cryptococcosis alongside the HIV/AIDS epidemic), only $4 \%-11 \%$ of patients with $C D 4^{+}$T-cell counts of less than $100 \mathrm{cells} / \mu \mathrm{l}$ in sub-Saharan Africa have cryptococcal antigen detectable in blood. ${ }^{25-34}$ This indicates that additional or underlying factors, in addition to $\mathrm{CD} 4^{+} \mathrm{T}$-cell deficiency, may lead to increased susceptibility in a subset of individuals. A greater understanding of host immunity to cryptococcosis has been achieved though recent studies in animal models as well as 
humans, both with and without immune-compromise. Research findings have established innate and acquired immune factors and genetic polymorphisms that influence an individual's ability to resist or survive infection with C. neoformans (reviewed in ${ }^{11,12}$ ). Cryptococcosis in patients in which no immune deficiency has been identified, such as in this case, may represent the existence of an underlying immunological or genetic predisposition.

\section{Treatment}

The Infectious Diseases Society of America (IDSA) recommends that apparently-immunocompetent patients (i.e. non-HIV and non-transplant recipients) with disseminated cryptococcosis are treated with high-dose amphotericin $B(0.7-1.0 \mathrm{mg} / \mathrm{kg}$ per day) and flucytosine ( $100 \mathrm{mg} / \mathrm{kg}$ per day in 4 divided doses) for up to 6 weeks followed by consolidation treatment with fluconazole (400 mg per day) for 8 weeks and maintenance low-dose fluconazole ( $200 \mathrm{mg}$ [3 mg/kg] per day) for 6-12 months. ${ }^{35}$ This approach to treatment is based on two early clinical trials in a heterogeneous group of patients with meningitis who were neither HIV-infected nor transplant recipients: the first trial documented the superiority of a combination low-dose amphotericin $B$ and high-dose flucytosine regimen vs low-dose amphotericin B alone; and, the second, the superiority of a 6-week vs 4-week combination regimen. ${ }^{36,37}$ These trials were published prior to the availability of triazole agents and adoption of the standard 3-phase regimen with a high-dose amphotericin $B$ and flucytosine backbone. Thus, the IDSA recommendations have been modified to include currently-accepted doses of amphotericin B deoxycholate and flucytosine and consolidation/ maintenance phases of treatment to reduce the risk of relapse. If flucytosine is not given or is unavailable, lengthening the duration of amphotericin B treatment by two weeks is recommended. ${ }^{35}$

\section{References}

1. Hagen F, Khayhan K, Theelen B, et al. Recognition of seven species in the Cryptococcus gattii/Cryptococcus neoformans species complex. Fungal Genet Biol. 2015 May;78:16-48.

2. Park BJ, Rajasingham $R$, Smith $R$, et al. Update on the global burden of cryptococcosis. Oral Abstract presented at: ICCC;Amsterdam; 2014;

3. Jarvis JN, Meintjes G, Williams A, et al. Adult meningitis in a setting of high HIV and TB prevalence: findings from 4961 suspected cases. BMC Infect Dis. 2010;10(1):67.

4. Britz E, Mollendorf C, von Gottberg A, et al. The epidemiology of bacterial and fungal meningitis among adults in Gauteng Province, 2009-2013 [Internet]. Oral Presentation presented at: FIDSSA; 2015 Nov; South Africa. Available from: http://www.mm3admin. co.za/documents/docmanager/47f686f5-acdf-4462-836664afd5633d81/00090269.pdf.

5. National Institute for Communicable Diseases. Surveillance report Cryptococcus species. GERMS-SA Annual Report 2015. NICD; 2015.

6. Pappas PG, Perfect JR, Cloud GA, et al. Cryptococcosis in human immunodeficiency virus-negative patients in the era of effective azole therapy. Clin Infect Dis. 2001 Sep;33(5):690-9.

7. Bratton EW, El Husseini N, Chastain CA, et al. Comparison and temporal trends of three groups with cryptococcosis: HIV-infected, solid organ transplant, and HIV-negative/non-transplant. In: Scheurer M, editor. PLoS ONE. 2012 Aug 24;7(8):e43582.

8. Lui G, Lee N, Ip $M$, et al. Cryptococcosis in apparently immunocompetent patients. QJM. 2006 Mar;99(3):143-51.

9. Mitchell DH, Sorrell TC, Allworth AM, et al. Cryptococcal disease of the CNS in immunocompetent hosts: influence of cryptococcal variety on clinical manifestations and outcome. Clin Infect Dis. 1995 Mar;20(3):611-6.

10. Meiring ST, Quan VC, Cohen C, et al. A comparison of cases of paediatric-onset and adult-onset cryptococcosis detected through population-based surveillance, 2005-2007. AIDS. 2012 Nov;26(18):2307-14.
11. Goldman DL, Khine $H$, Abadi J, et al. Serologic evidence for Cryptococcus neoformans infection in early childhood. Pediatrics. 2001 May; 107(5):e66.

12. Deshaw M, Pirofski LA. Antibodies to the Cryptococcus neoformans capsular glucuronoxylomannan are ubiquitous in serum from HIV+ and HIV- individuals. Clin Exp Immunol. 1995 Mar;99(3):425-32.

13. Fleuridor R, Lyles RH, Pirofski L. Quantitative and qualitative differences in the serum antibody profiles of human immunodeficiency virusinfected persons with and without Cryptococcus neoformans meningitis. J Infect Dis. 1999 Nov;180(5):1526-35.

14. Abadi J, Pirofski L. Antibodies reactive with the cryptococcal capsular polysaccharide glucuronoxylomannan are present in sera from children with and without human immunodeficiency virus infection. J Infect Dis. 1999 Sep;180(3):915-9.

15. Garcia-Hermoso D, Janbon G, Dromer F. Epidemiological evidence for dormant Cryptococcus neoformans infection. J Clin Microbiol. 1999 Oct;37(10):3204-9.

16. Ma H, May R. Virulence in Cryptococcus species. In: Advances in applied microbiology. Burlington, VT: Academic Press; 2009. p. 131-90.

17. May RC, Stone NRH, Wiesner DL, et al. Cryptococcus: from environmental saprophyte to global pathogen. Nat Rev Microbiol. 2015 Dec 21;14(2):106-17.

18. Rohatgi S, Pirofski L. Host immunity to Cryptococcus neoformans. Future Microbiol. 2015 Apr;10(4):565-81.

19. Coelho C, Bocca AL, Casadevall A. The intracellular life of Cryptococcus neoformans. Annu Rev Pathol Mech Dis. 2014 Jan 24;9(1):219-38.

20. Steenbergen JN, Shuman HA, Casadevall A. Cryptococcus neoformans interactions with amoebae suggest an explanation for its virulence and intracellular pathogenic strategy in macrophages. Proc Natl Acad Sci USA. 2001 Dec 18;98(26):15245-50.

21. Tucker SC, Casadevall A. Replication of Cryptococcus neoformans in macrophages is accompanied by phagosomal permeabilization and accumulation of vesicles containing polysaccharide in the cytoplasm. Proc Natl Acad Sci USA. 2002 Mar 5;99(5):3165-70.

22. Casadevall A, Steenbergen JN, Nosanchuk JD. 'Ready made' virulence and 'dual use' virulence factors in pathogenic environmental fungi -the Cryptococcus neoformans paradigm. Curr Opin Microbiol. 2003 Aug;6(4): 332-7.

23. Kapoor A, Flechner SM, O'Malley K, et al. Cryptococcal meningitis in renal transplant patients associated with environmental exposure. Transpl Infect Dis. 1999 Sep;1(3):213-7.

24. Nosanchuk JD, Shoham S, Fries BC, et al. Evidence of zoonotic transmission of Cryptococcus neoformans from a pet cockatoo to an immunocompromised patient. Ann Intern Med. 2000 Feb 1;132(3):205-8

25. Desmet $\mathrm{P}$, kayembe $\mathrm{K}$, Vroey $\mathrm{C}$. The value of cryptococcal serum antigen screening among HIV-positive/AIDS patients in Kinshasa, Zaire. AIDS. 1989;3(2):77-8.

26. French N, Gray K, Watera C, et al. Cryptococcal infection in a cohort of HIV-1-infected Ugandan adults. AIDS 2002 May 3;16(7):1031-8.

27. Jarvis JN, Lawn SD, Vogt $M$, et al. Screening for cryptococcal antigenemia in patients accessing an antiretroviral treatment program in South Africa. Clin Infect Dis. 2009 Apr;48(7):856-62.

28. Lara-Peredo O, Cuevas LE, French N, et al. Cryptococcal infection in an HIV-positive Ugandan population. J Infect. 2000 Sep;41(2):195.

29. Liechty CA, Solberg P, WereW, et al. Asymptomatic serum cryptococcal antigenemia and early mortality during antiretroviral therapy in rural Uganda. Trop Med Int Health TM IH. 2007 Aug;12(8):929-35.

30. Longley N, Jarvis JN, Meintjes G, et al. Cryptococcal antigen screening in patients initiating ART in South Africa: a prospective cohort study. Clin Infect Dis. 2016 Mar 1;62(5):581-7.

31. Meyer $A-C L$, Kendi $C K$, Penner JA, et al. The impact of routine cryptococcal antigen screening on survival among HIV-infected individuals with advanced immunosuppression in Kenya. Trop Med Int Health. 2013 Apr;18(4):495-503.

32. Osazuwa F, Dirisu JO, Okuonghae PE, et al. Screening for cryptococcal antigenemia in anti-retroviral naïve AIDS patients in Benin City, Nigeria. Oman Med J. 2012 May 16;27(3):228-31.

33. Oyella J, Meya D, Bajunirwe F, et al. Prevalence and factors associated with cryptococcal antigenemia among severely immunosuppressed HIV-infected adults in Uganda: a cross-sectional study. J Int AIDS Soc. 2012;15(1):15. 
34. Rugemalila J, Maro VP, Kapanda G, et al. Cryptococcal antigen prevalence in HIV-infected Tanzanians: a cross-sectional study and evaluation of a point-of-care lateral flow assay. Trop Med Int Health. 2013 Sep; 18(9):1075-9.

35. Perfect JR, Dismukes WE, Dromer F, et al. Clinical practice guidelines for the management of cryptococcal disease: 2010 update by the Infectious Diseases Society of America. Clin Infect Dis. 2010 Feb;50(3):291-322.
36. Bennett JE, Dismukes WE, Duma RJ, et al. A comparison of amphotericin $B$ alone and combined with flucytosine in the treatment of cryptoccal meningitis. N Engl J Med. 1979 Jul 19;301(3):126-31.

37. Dismukes WE, Cloud G, Gallis HA, et al. Treatment of cryptococcal meningitis with combination amphotericin $B$ and flucytosine for four as compared with six weeks. N Engl J Med. 1987 Aug 6;317(6):334-41.

Received: 09-05-2016 Accepted: 09-05-2016 